\title{
Effect of $x$-ray energy on the radiological image quality in propagation-based phase-contrast computed tomography of the breast
}

\author{
Sarina Wan $\odot,{ }^{a} *$ Benedicta D. Arhatari, ${ }^{\text {b,c }}$ Yakov I. Nesterets $\odot, \stackrel{\text { d,e }}{ }$ \\ Sheridan C. Mayo, ${ }^{\mathrm{d}}$ Darren Thompson $\odot,{ }^{\text {d,e }}$ Jane Fox, ${ }^{\text {f,g }}$ Beena Kumar, ${ }^{\mathrm{g}}$ \\ Zdenka Prodanovic, ${ }^{g}$ Daniel Hausermann, ${ }^{b}$ Anton Maksimenko, ${ }^{b}$ \\ Christopher Hall $\odot,{ }^{b}$ Matthew Dimmock $\odot,{ }^{f}$ Konstantin M. Pavlov $\odot, ~, h, i$ \\ Darren Lockie, ${ }^{j}$ Mary Rickard, ${ }^{\text {a Ziba Gadomkar, }},{ }^{\text {Alaleh Aminzadeh } \odot, ~}$ \\ Elham Vafa, ${ }^{\text {a }}$ Andrew Peele, ${ }^{\mathrm{b}}$ Harry M. Quiney, ${ }^{\mathrm{c}}$ Sarah Lewis, \\ Timur E. Gureyev, ${ }^{\text {a,c,e,i }}$ Patrick C. Brennan ${ }^{\circ},{ }^{a}$ and \\ Seyedamir Tavakoli Taba ${ }^{\text {a }}$ \\ ${ }^{a}$ University of Sydney, Faculty of Medicine and Health, Department of Medical Radiation \\ Sciences, Lidcombe, Australia \\ ${ }^{\mathrm{b}}$ Australian Synchrotron, ANSTO, Clayton, Australia \\ 'University of Melbourne, School of Physics, Parkville, Australia \\ ${ }^{\mathrm{d} C}$ Commonwealth Scientific and Industrial Research Organisation, Clayton, Australia \\ ${ }^{e}$ University of New England, School of Science and Technology, Armidale, Australia \\ ${ }^{\mathrm{f}}$ Monash University, Faculty of Medicine, Nursing and Health Sciences, Clayton, Australia \\ ${ }^{g}$ Monash Health, Department of Pathology, Clayton, Australia \\ ${ }^{\text {h}}$ University of Canterbury, School of Physical and Chemical Sciences, Christchurch, New Zealand \\ iMonash University, School of Physics and Astronomy, Clayton, Australia \\ ${ }^{\mathrm{j}}$ Maroondah BreastScreen, Eastern Health, Ringwood, Australia
}

\begin{abstract}
Purpose: Breast cancer is the most common cancer in women in developing and developed countries and is responsible for $15 \%$ of women's cancer deaths worldwide. Conventional absorption-based breast imaging techniques lack sufficient contrast for comprehensive diagnosis. Propagation-based phase-contrast computed tomography (PB-CT) is a developing technique that exploits a more contrast-sensitive property of $\mathrm{x}$-rays: $\mathrm{x}$-ray refraction. X-ray absorption, refraction, and contrast-to-noise in the corresponding images depend on the x-ray energy used, for the same/fixed radiation dose. The aim of this paper is to explore the relationship between $\mathrm{x}$ ray energy and radiological image quality in PB-CT imaging.
\end{abstract}

Approach: Thirty-nine mastectomy samples were scanned at the imaging and medical beamline at the Australian Synchrotron. Samples were scanned at various x-ray energies of 26, 28, 30, 32, 34, and $60 \mathrm{keV}$ using a Hamamatsu Flat Panel detector at the same object-to-detector distance of $6 \mathrm{~m}$ and mean glandular dose of $4 \mathrm{mGy}$. A total of 132 image sets were produced for analysis. Seven observers rated PB-CT images against absorption-based CT (AB-CT) images of the same samples on a five-point scale. A visual grading characteristics (VGC) study was used to determine the difference in image quality.

Results: PB-CT images produced at 28, 30, 32, and $34 \mathrm{keV}$ x-ray energies demonstrated statistically significant higher image quality than reference AB-CT images. The optimum x-ray energy, $30 \mathrm{keV}$, displayed the largest area under the curve $\left(\mathrm{AUC}_{\mathrm{VGC}}\right)$ of $0.754(p=0.009)$. This was followed by $32 \mathrm{keV}\left(\mathrm{AUC}_{\mathrm{VGC}}=0.731, p \leq 0.001\right), 34 \mathrm{keV}\left(\mathrm{AUC}_{\mathrm{VGC}}=0.723\right.$, $p \leq 0.001)$, and $28 \mathrm{keV}\left(\mathrm{AUC}_{\mathrm{VGC}}=0.654, p=0.015\right)$.

Conclusions: An optimum energy range (around $30 \mathrm{keV}$ ) in the PB-CT technique allows for higher image quality at a dose comparable to conventional mammographic techniques. This results in improved radiological image quality compared with conventional techniques, which may ultimately lead to higher diagnostic efficacy and a reduction in breast cancer mortalities.

*Address all correspondence to Sarina Wan, sarina.wan@gmail.com 
Keywords: phase-contrast CT; propagation-based phase-contrast imaging; breast cancer diagnosis; breast cancer; X-ray energies.

Paper 21028SSRR received Feb. 6, 2021; accepted for publication Jun. 28, 2021; published online Jul. 12, 2021.

\section{Introduction}

Every year, around $25 \%$ of new cancer cases detected in women are breast cancer. ${ }^{1}$ Breast cancer also accounts for $15 \%$ of female cancer mortalities in developing and developed countries. ${ }^{2}$ The early detection of breast cancer is pivotal for decreasing associated mortalities. Breast screening facilitates the early detection of breast cancer, which makes treatment more successful. The Australian Institute of Health and Welfare ${ }^{3}$ found that breast cancer mortalities reduced from 74 deaths per 100,000 women aged 50 to 74 in 1991, when screening began, to <40 deaths per 100,000 women in 2018 (a reduction of almost 46\%).

Digital mammography (DM) is the most common modality used for breast screening ${ }^{4}$ and diagnostic imaging of symptomatic patients: over 50\% of cancer cases in Australia are diagnosed outside of the BreastScreen program, and all would have diagnostic mammography. ${ }^{3}$ Apart from its low dose and cost, DM can reduce breast cancer mortality rates by allowing for early detection and treatment of disease. However, although DM is efficient, major weaknesses limit its diagnostic accuracy. Even though DM provides the highest spatial resolution of current modalities, the nature of two-dimensional images results in superimposition of breast tissue. ${ }^{5}$ The sensitivity values for DM range from $72 \%$ to $90.6 \%$, while the specificity values span from $72 \%$ to $98 \%$, signifying a margin for improved pathology detection. ${ }^{6-11}$ In addition, the efficacy of DM may vary across individuals, depending on their breast density. ${ }^{8,12}$ Mandelson et al. ${ }^{8}$ discovered that sensitivity could be as low as $30 \%$ in extremely dense breasts. Digital breast tomosynthesis (DBT) can assist with the issue of overlapping structures by acquiring a series of projection images at different angles and producing images for different depths in the breast. ${ }^{5,13} \mathrm{Phi}$ et al. ${ }^{10}$ found that the sensitivity of DM and DBT combined to be $84 \%$ to $90 \%$, which exceeded the sensitivity of $72 \%$ in DM alone. Despite the advantages, patients who undergo DBT and DM examinations together receive higher radiation doses than undertaking one examination alone. In addition, breast compression may cause patients to experience discomfort and pain; however, it is necessary for decreasing breast thickness, increasing soft tissue uniformity, and decreasing dose. ${ }^{14,15}$ The use of suboptimal compression in patients unable to cope with the discomfort may lead to reduced contrast resolution in the image. Breast $\mathrm{CT}$ also overcomes superimposition by providing three-dimensional (3D) images. While CT increases visualization of mass lesions and edges compared with DM, ${ }^{16,17}$ it has substantially higher radiation dose levels and lower spatial resolution compared with DM. ${ }^{17}$ Lindfors et al. ${ }^{16}$ noted that breast CT is more physically comfortable than DM and DBT as no compression is required.

The above conventional $\mathrm{x}$-ray imaging techniques are established on the basis of radiation absorption. ${ }^{18}$ An x-ray from a source passes through an object and is attenuated according to the object's density, forming contrast in the image. However, as the breast comprises tissues with similar densities, the contrast in the x-ray absorption images is minimal, making it difficult to distinguish pathology from healthy tissue due to dose restrictions. ${ }^{12,19}$ Furthermore, insufficient contrast-to-noise increases the chances of misinterpreting benign from malignant tumors, which may affect the treatment pathway of a patient. Thus, understanding the disadvantages of current $\mathrm{x}$-ray imaging modalities allows for better insight into the evolution of new technologies that may improve breast cancer diagnosis and treatment.

Phase-contrast imaging is a well-developed technology that aims to address the existing limitations of current absorption-based methods. The phase-contrast techniques utilize another type of interaction between x-rays and imaged objects, namely refraction. The behavior of x-rays when they travel through an object can be described using the complex refractive index,

2329-4302/2021/\$28.00 (C) 2021 SPIE 
$n=1-\delta+i \beta$, where $\delta$ incorporates refractive effects and $\beta$ describes absorption. ${ }^{20,21}$ The refraction term $(\delta)$ has been found to be up to a thousand times larger than the absorption term $(\beta)$ at typical mammographic energies of 15 to $25 \mathrm{keV},{ }^{22,23}$ and thus, the refraction of $\mathrm{x}$-rays results in additional contrast in projection images that is usually greater than absorption contrast.

Although there are many phase-contrast imaging techniques such as analyzer-based imaging, crystal interferometry, grating interferometry, and edge illumination, propagation-based imaging (PBI) may provide benefits while having the most straightforward approach. ${ }^{24}$ In PBI, components of the imaging setup are placed in a straight line, requiring no special optical elements between the source, object, and detector. PBI closely resembles the setup of conventional x-ray imaging, with the exception of an increased object-to-detector distance to exploit refraction effects and a requirement for a spatially coherent x-ray illumination. ${ }^{25}$ Consequently, a highly spatially coherent beam allows phases of the beam to develop interference patterns, particularly highlighting edges of the object. ${ }^{22}$ Furthermore, when combined with $\mathrm{CT}$, known as propagation-based CT (PB-CT), a detailed 3D representation of the breast is obtained at a dose comparable to DM. ${ }^{26,27}$ Since compression is also not required in PB-CT, comfort to patients is increased without compromising the image quality or radiation dose. Earlier studies have found that PB-CT of soft tissues may provide a 10-fold or even higher increase in signal-to-noise ratio and contrast-to-noise ratio (CNR) in comparison with conventional absorption-based CT (ABCT) ${ }^{28,29}$ This increases the probability of better visualizing mass lesions and anatomical edges as well as providing superior image quality to identify smaller lesions previously undetectable in absorption-based methods. Furthermore, the differences between benign and malignant features may become more distinct due to lower noise, increased soft-tissue contrast, and better edge definition in PB-CT compared with AB-CT. ${ }^{26}$ Therefore, PB-CT may provide a solution to balancing high spatial and contrast resolution at an acceptable radiation dose, ultimately leading to increased diagnostic accuracy.

For successful translation of this technology to the medical imaging domain, further research needs to be conducted on optimization factors that determine image quality in PB-CT. Such optimization factors include the choice of x-ray energy, source-to-detector distance, use of maximum intensity projection, CT reconstruction method, phase-retrieval algorithm, detector type, and pixel size. ${ }^{30,31}$ In particular, $\mathrm{x}$-ray energy has always been a fundamental component in determining the image quality of established imaging methods. Traditionally, mammographic breast imaging requires relatively low $\mathrm{x}$-ray energies to maximize image contrast. ${ }^{32} \mathrm{~A}$ higher energy would result in increased penetration of x-rays (i.e., higher transparency of the tissues), ultimately decreasing the image contrast while increasing the relative weight of Compton scattering in attenuating x-rays. Lower energies allow for greater differences in gray levels to be perceived, with only photons with enough energy reaching the detector. However, using $\mathrm{x}$-ray energies that are too low results in an increased radiation dose to the patient as X-ray photons with insufficient energy are completely absorbed by patient tissue and do not reach the detector. Thus, these photons do not contribute to the image contrast. ${ }^{32}$

There is limited research specifically underlining the most suitable x-ray energy for PB-CT, and the work that has been done has produced varying results. Recent studies by Oliva et al. ${ }^{33}$ and Delogu et al. ${ }^{34}$ discovered an optimum energy range between 26 and $28 \mathrm{keV}$ for an ideal detector. Some studies claim that energies of around 32 to $35 \mathrm{keV}$ are most appropriate, ${ }^{30,31}$ but these studies have not examined x-ray energies lower than $32 \mathrm{keV}$. On the other hand, Ghani et al. ${ }^{35}$ and Zhao et al. ${ }^{26}$ indicated that a higher peak energy- $120 \mathrm{kVp}$, equivalent to an effective beam energy of about $60 \mathrm{keV}$ - can increase penetration, leading to a decreased scan time and dose. However, Ghani et al. ${ }^{35}$ did not incorporate CT with a phase contrast technique, and Zhao et al. ${ }^{26}$ implemented $60 \mathrm{keV}$ with analyzer-based imaging, both of which may not directly correspond to PB-CT. Therefore, a deeper understanding of the relationship between X-ray energy and radiological image quality is required to progress the PB-CT technique for breast cancer imaging. The aim of this paper is to investigate the optimum $\mathrm{x}$-ray energy required to produce the highest radiological image quality in PB-CT based on a human observer study. 


\section{Materials and Methods}

\subsection{Samples and Imaging Techniques}

The Humans Research Ethics Committee of Monash University (project number: CF15/31382015001340) approved imaging breast mastectomy samples for experimental purposes. All patients provided written consent for the use of the specimens. All images were de-identified before being used in this study. The majority of mastectomies possessed malignant tumors, while some were cancer free or only contained benign lesions. Table 1 provides detailed descriptions of the breast pathology.

The mastectomy samples were scanned at the Imaging and Medical beamline (IMBL) of the Australian Synchrotron. After the surgery, the mastectomies were placed in a plastic low-absorbing cylindrical container with an 11-cm diameter to be imaged. The container was then placed on a rotating stage for scanning. The breast samples included in this study comprised those that had been scanned at multiple x-ray energies. However, not all samples were scanned at all energies, mainly due to beamtime limitations and equipment failure in some cases. Samples were excluded if they were not standard size, meaning a container with a diameter larger or smaller than $11 \mathrm{~cm}$ was used. After the samples had been filtered, 39 samples were included in the study, producing a total of 132 image sets at various x-ray energies (Table 2).

The images were produced using a Hamamatsu C10900D Flat Panel Sensor that had a fieldof-view of $1216 \times 1232$ pixels, a pixel size of $100 \mu \mathrm{m} \times 100 \mu \mathrm{m}$, and a frame rate of 17 frames per second. For each scan at $4 \mathrm{mGy}, 2400$ projections with 0.075 deg angular steps were collected over a 180-deg rotation angle, and each scan took around $141 \mathrm{~s}$. The reconstruction method used was filtered backprojection. PB-CT images were scanned with the detector placed at the maximum achievable distance of $6 \mathrm{~m}$ from the sample (at the IMBL). Furthermore, this study applied a phase-retrieval technique to the phase-contrast images through the homogeneous transport of intensity equation (TIE-Hom) algorithm. ${ }^{36}$ In full-phase retrieval, a $\delta / \beta$ ratio (in the complex refraction index) equal to the theoretical value of glandular tissue relative to blood is used. It should be noted that the relative $\delta / \beta$ for glandular and cancerous (blood) tissues is

Table 1 Number of breast mastectomy samples per lesion type.

\begin{tabular}{lc}
\hline \hline Lesion type & No. of samples per lesion type \\
\hline No invasive carcinoma & 22 \\
No invasive tumor & 4 \\
Invasive carcinoma & 22 \\
No DCIS & 1 \\
Low grade DCIS & 8 \\
Intermediate grade DCIS & 5 \\
High grade DCIS & 2 \\
BRE grade 1 & 10 \\
BRE grade 2 & 1 \\
BRE grade 3 & 7 \\
No special type & 1 \\
Mixed ductal and lobular features & 3 \\
\hline LCIS & 2 \\
\hline Note: BRE, BloOm Richardson-EIston & 13 \\
\hline
\end{tabular}

Note: BRE, Bloom-Richardson-Elston system; DCIS, ductal carcinoma in situ; LCIS, lobular carcinoma in situ. 
Wan et al.: Effect of $\mathrm{x}$-ray energy on the radiological image quality in propagation-based...

Table 2 Number of scanned samples per x-ray energy.

\begin{tabular}{lc}
\hline \hline Energy $(\mathrm{keV})$ & No. of samples scanned per energy \\
\hline 26 & 9 \\
28 & 25 \\
30 & 9 \\
32 & 38 \\
34 & 39 \\
60 & 12 \\
\hline \hline
\end{tabular}

smaller than the relative $\delta / \beta$ for glandular and adipose tissues. Thus, selecting these values maximizes the differentiation of glandular and cancerous tissues. Half-phase retrieval uses a value that is half of the full-phase retrieval value. In this study, half-phase retrieval was applied as higher quality images were demonstrated using this method compared with full-phase retrieval, as determined by a previous study using the same detector. ${ }^{37}$ The mean glandular dose (MGD) received by the samples was $4 \mathrm{mGy}$, which may represent the radiation dose in two-view DM. The radiation dose received by the samples was determined through a Monte Carlo simulation in which we used a numerical breast phantom composed of $30 \%$ glandular tissue and $70 \%$ adipose tissue, surrounded by a 5-mm thick layer of adipose tissue to represent the skin. Further details about the MGD calculation method used in this study has been described in our previous publications. ${ }^{30,38}$

The PB-CT slices were originally reconstructed in the coronal view. The slices were then converted into axial and sagittal views to mimic the craniocaudal (CC) and mediolateral oblique views, respectively, reflecting traditional projections used in mammographic breast imaging. Each set contained around a thousand CT slices with $100 \mu \mathrm{m}$ thickness. To reduce the assessment time, slices with the thickness of $1 \mathrm{~mm}$ were produced by resampling every 10 slices using maximum intensity projection, while preserving the in-plane resolution of $100 \mu \mathrm{m}$.

The energy ranges used to obtain PB-CT images included 26, 28, 30, 32, 34, and $60 \mathrm{keV}$. For each sample, the PB-CT images at various energies were compared with AB-CT images of the same sample. All AB-CT images were scanned at $32 \mathrm{keV}$ (this energy was shown to provide high quality AB-CT images in some of our previous studies) ${ }^{27,37,39}$ and using the same parameters as PB-CT images, except that they were scanned with the minimum achievable sample-to-detector distance of $0.19 \mathrm{~m}$ and no phase-retrieval was applied (since the AB-CT images do not contain phase contrast). The dose delivered for AB-CT images was also $4 \mathrm{mGy}$.

\subsection{Image Assessment}

\subsubsection{Image preparation}

Final images matching the inclusion criteria were selected. The background of all images was segmented out using a manual threshold. The axial and sagittal view images were converted to digital imaging and communications in medicine format and then loaded onto a mammography workstation. Next, images were manually synched, so assessors could view and scroll through the test (PB-CT) images and reference (AB-CT) images simultaneously.

Data collection was completed in a 1-month time period for the convenience of assessors and researchers. Assessors included seven medical imaging experts in medical physics and diagnostic radiography with 4 to 30 years of experience in diagnostic imaging. Assessors were blinded of the imaging conditions, which included the energies and the image type-PB-CT or AB-CTused in each comparison. The assessors were permitted to evaluate the images at their own pace with no time limit. The average time taken to complete all of the image sets was approximately one and a half hours for each assessor. 


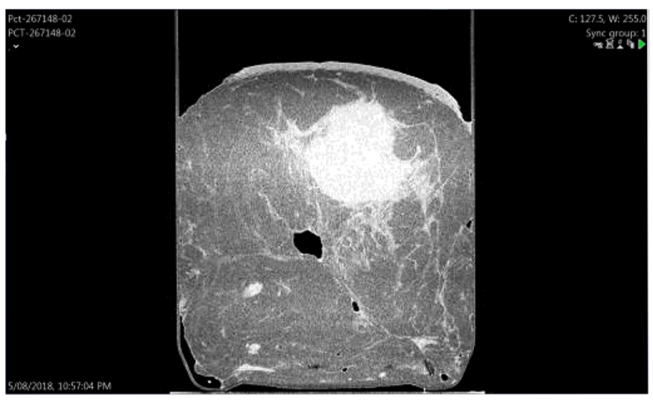

(a)

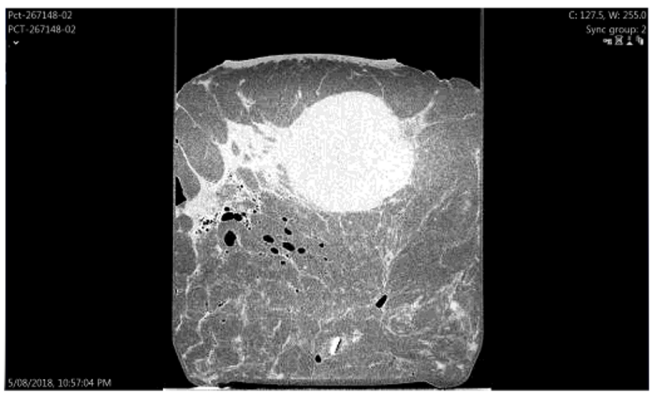

(c)

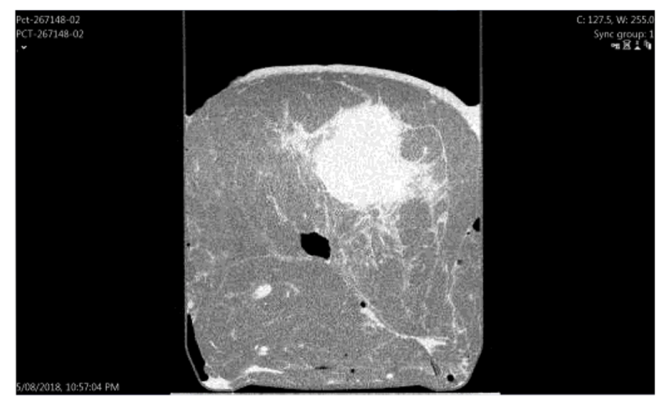

(b)

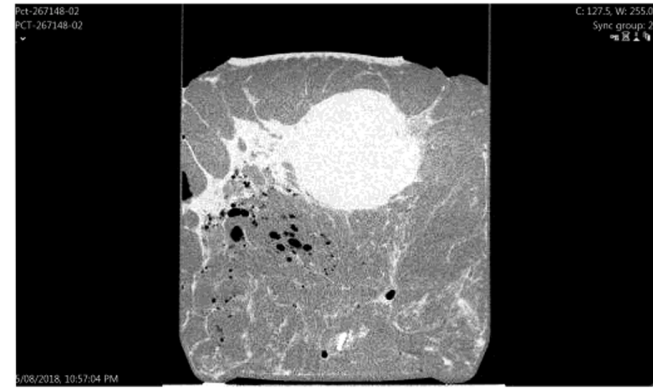

(d)

Fig. 1 Screen setup of PB-CT versus AB-CT images. This case was a post neoadjuvant chemotherapy of invasive carcinoma, no special type, no residual in-situ, or invasive disease (sample ID: 267148); (a) axial PB-CT slice; (b) axial AB-CT slice; (c) sagittal PB-CT slice; (d) sagittal AB-CT slice; PB-CT images for this image set were scanned at an x-ray energy of $28 \mathrm{keV}$; AB-CT images were scanned at an x-ray energy of $32 \mathrm{keV}$.

The reading environment and screen on which the images were viewed was controlled for all participants. Two 5MP mammographic reporting monitors divided into four sections were used. The left column displayed the test images, and the right column presented the reference images. The top row displayed the axial slices, while the bottom row displayed the sagittal slices, as seen in Fig. 1.

\subsubsection{Radiological assessment}

The image quality assessment was carried out in the form of a visual grading characteristic (VGC) study. ${ }^{40}$ Image quality is essentially determined by the experts who are interpreting the image, and opinions may vary between individuals. To assess the correlation between variables, expert opinion needs to be measured in a way that expresses their opinions quantitively. VGC studies are high in practical relevance as the tests reflect a clinician's confidence in identifying structures and pathologies often seen in the clinical environment, particularly for breast imaging. 41

An assessment sheet with instructions and a scoring table was designed and provided to the assessors. Images were evaluated via a five-point rating scale comparing the test image with the reference image to reflect the assessor's opinion on image quality. The definition of the scoring system is given in Table 3. Assessors were also asked to focus specifically on six attributes relating to the radiological image quality, given in Table 4. The images contained pathologies consisting of malignant or benign features, and others possessed cancer-free lesions to mimic the clinical environment. After the assessors had completed the assessment, the collected data were imported to excel for further analysis.

\subsection{Data Analysis}

An interobserver agreement was calculated via the intraclass correlation coefficient (ICC) to examine the reliability of the data collected from the assessors. The ICC determines the amount 
Wan et al.: Effect of $\mathrm{x}$-ray energy on the radiological image quality in propagation-based...

Table 3 Five-point rating scale comparing test image with reference image.

\begin{tabular}{ll}
\hline \hline Score & \multicolumn{1}{c}{ Description } \\
\hline+2 & Image quality is exceptionally better than the reference image \\
+1 & Image quality is slightly better than the reference image \\
0 & Equal image quality to the reference image \\
-1 & Image quality is slightly worse than the reference image \\
-2 & Image quality is exceptionally worse than the reference image \\
\hline \hline
\end{tabular}

Table 4 Attributes contributing to radiological quality.

\begin{tabular}{ll}
\hline \hline Attribute & \multicolumn{1}{c}{ Description } \\
\hline Perceptible contrast & Difference between low and high radiolucency in various soft tissue regions \\
Lesion sharpness & Clarity of definition of lesions and spiculations. \\
Normal tissue interfaces & Clarity of visualization of interfaces between fatty and fibro-glandular tissues \\
Calcification visibility & Sharpness of microcalcifications (if any) \\
Image noise & Presence of quantum mottle in the image \\
Artifacts & Evidence of any other technical artefacts such as rings or distortions \\
\hline \hline
\end{tabular}

of agreement between different units or individuals. ${ }^{42}$ Calculating the ICC is important in this study because image quality assessment is essentially a subjective task, with possible variations between assessors. The ICC form used was a two-way random with an average of multiple readers, also conventionally represented as ICC $(2, k)$. This form generalizes the reliability results to a larger population of assessors with the same characteristics as the selected assessors. ${ }^{43}$ The ICC was implemented before data analysis to ensure that no outliers were present. The ICC was calculated through the IBM SPSS Statistics (Version 27.0) software. ${ }^{44}$

As the scales in VGC studies were ordinal, a nonparametric rank-invariant test was used to analyze data and measure the variance between different energies. The raw data collected from the assessments was analyzed via the VGC Analyzer Software (Version 1.0.2).$^{40}$ An area under the curve $\left(\mathrm{AUC}_{\mathrm{VGC}}\right)$ was calculated from the cumulative distribution of quality scores of test images against corresponding reference images. In doing so, we applied bootstrapping with 2000 resamples of rating scores. ${ }^{40}$ The $\mathrm{AUC}_{\mathrm{VGC}}$ provided a relative measure in the difference in image quality between two image sets. In our case, we compared the PB-CT test condition against the $\mathrm{AB}-\mathrm{CT}$ reference condition. A $p$-value was calculated from the $\mathrm{AUC}_{\mathrm{VGC}}$, with a $5 \%$ significance level and a $95 \%$ confidence interval.

\section{Results}

Interobserver agreement was calculated through the ICC. In this case, the ICC examines how similarly the seven assessors rated the radiological quality of the test images compared with the reference images. The ICC was calculated to be 0.867 . The $95 \%$ confidence interval of the ICC had a lower bound of 0.826 and an upper bound of 0.897 .

An ICC score of $<0.500$ represents poor agreement; an interval between 0.500 and 0.750 demonstrates a fair agreement; scores between 0.750 and 0.900 indicate good agreement; and scores between 0.900 and 1.00 reflect excellent agreement. ${ }^{43}$ The calculated ICC results indicate good inter-rater reliability, and no assessors were excluded from the data analysis. 
Wan et al.: Effect of $x$-ray energy on the radiological image quality in propagation-based...

Table 5 Data analysis of the $\mathrm{AUC}_{\mathrm{VGC}}$ resulting from the $\mathrm{VGC}$ analysis for PB-CT images versus AB-CT images.

\begin{tabular}{lcccccc}
\hline \hline & & & & \multicolumn{2}{c}{$95 \%$ confidence interval } & \\
\cline { 5 - 6 } & & & & & & \\
& 26 & 9 & 0.454 & 0.278 & 0.627 & 0.510 \\
& 28 & 25 & $0.654^{*}$ & 0.571 & 0.734 & 0.015 \\
PB-CT versus & 30 & 9 & $0.754^{*}$ & 0.667 & 0.849 & 0.009 \\
AB-CT at 4 mGy & 32 & 38 & $0.731^{*}$ & 0.675 & 0.788 & $\leq 0.001$ \\
& 34 & 39 & $0.723^{*}$ & 0.668 & 0.771 & $\leq 0.001$ \\
& 60 & 12 & $0.162^{*}$ & 0.060 & 0.298 & 0.009 \\
\hline \hline
\end{tabular}

${ }^{*} p \leq 0.05$

\subsection{Visual Grading Analysis}

Table 5 shows bootstrapped $\mathrm{AUC}_{\mathrm{VGC}}$ values, $95 \%$ confidence intervals, and $p$-values for PB-CT in comparison with AB-CT images. Comparing the test and reference conditions, images produced with energies of $28 \mathrm{keV}(p=0.015), 30 \mathrm{keV}(p=0.009), 32 \mathrm{keV}(p \leq 0.001)$, and $34 \mathrm{keV}(p \leq 0.001)$ had higher radiological quality than the reference condition. For comparison, PB-CT images corresponding to $26 \mathrm{keV}$ (not significantly) and $60 \mathrm{keV}$ ( $p=0.009$ ) had lower radiological quality than the reference image. This is indicated by the $\mathrm{AUC}_{\mathrm{VGC}}$ in which a score above 0.500 reflects a higher quality than the reference state. Moreover, a score of 0.500 indicates the same image quality as the reference image, and a score between 0.00 and 0.500 depicts the test condition having a lower radiological quality than the reference condition. ${ }^{40}$ At the $\mathrm{x}$-ray energies of $28,30,32,34$, and $60 \mathrm{keV}$, the test images were significantly different from the reference images, as indicated by their $p$-values. An x-ray energy of $30 \mathrm{keV}$ produced the highest $\mathrm{AUC}_{\mathrm{VGC}}$ value of 0.754 .

\section{Discussion}

The findings demonstrate that, at particular x-ray energies, PB-CT of mastectomy specimens provides a higher radiological image quality than AB-CT. In this study, PB-CT images scanned at the energies of $28,30,32$, and $34 \mathrm{keV}$ produced significantly superior image quality compared with reference images scanned with $\mathrm{AB}-\mathrm{CT}$. The energy that displayed the largest $\mathrm{AUC}_{\mathrm{VGC}}$, and therefore the highest radiological image quality, was $30 \mathrm{keV}$. This was followed by 32, 34, and $28 \mathrm{keV}$, respectively. However, PB-CT images taken at 26 and $60 \mathrm{keV}$ did not present higher image quality than the AB-CT images captured at $32 \mathrm{keV}$, based on their $\mathrm{AUC}_{\mathrm{VGC}}$ values of 0.454 and 0.162 , respectively.

There is a narrow "band" of energies used in conventional mammography that can maximize the CNR. Sufficient CNR provides the image with increased contrast, so greater detail can be observed. ${ }^{45}$ Similarly, in PB-CT, an optimum energy can minimize the amount of noise perceived in an image at an acceptable radiation dose. Oliva et al. ${ }^{33}$ recently concluded that an optimum energy range of 26 to $28 \mathrm{keV}$ produced the highest CNR, complementing the results of Delogu et al., ${ }^{34}$ who discovered that the highest quality images were created by $28 \mathrm{keV}$ x-rays. However, these two studies applied a different detector, namely Pixirad-8 photon-counting detector with $60 \mu \mathrm{m}$ pitch in a honeycomb matrix, from the one used in this study. On the other hand, Tavakoli Taba et al. ${ }^{37}$ used the same Hamamatsu detector utilized in this study as well as 12 mastectomy samples and found that, for two x-ray energies of 32 and $34 \mathrm{keV}$, PB-CT resulted in higher image quality than AB-CT. The Hamamatsu C10900D Flat Panel Sensor is optimized for energies between 20 and $90 \mathrm{kVp} .{ }^{46}$ Baran et al. $^{30}$ and Tavakoli Taba et al. ${ }^{31}$ also investigated xray energies (specifically 32,35 , and $38 \mathrm{keV}$ ) along with other imaging parameters such as 
Wan et al.: Effect of $\mathrm{x}$-ray energy on the radiological image quality in propagation-based...

sample-to-detector distance, reconstruction method, and level of phase-retrieval using one mastectomy sample and showed that $32 \mathrm{keV}$ was generally superior to higher energies. This paper, however, solely focused on optimizing x-ray energy, which allowed us to utilize a larger number of samples (the largest number of mastectomy samples used in a PB-CT optimization study so far) and a wider range of $\mathrm{x}$-ray energies.

In conventional mammography, low energies do not have enough power to penetrate through the entire breast, resulting in an increase in radiation dose. On the other hand, excessive energy levels cause the resulting x-ray image to display inferior contrast, through a reduction in the differences in gray levels. In conventional mammography, Tomal et al. ${ }^{47}$ discovered that the optimum peak voltage of the $\mathrm{x}$-ray source is in the range of 23 to $30 \mathrm{kVp}$. Our study demonstrates that the same rules are applicable to PB-CT of the breast, where a band of $\mathrm{x}$-ray energies between 28 and $34 \mathrm{keV}$, and not necessarily one particular energy, may provide the most optimal images. In particular, the image quality is maximized at x-ray energies around 30 and $32 \mathrm{keV}$. Figure 2 displays the differences in image quality between a PB-CT image scanned at $30 \mathrm{keV}$ with its respective AB-CT reference slice (sample ID: 323592).

Higher energies, including $60 \mathrm{keV}$, have the potential to decrease the dose received by the patient as most X-rays will reach the detector and exit the body. However, that would be at the cost of a significantly lower CNR in PB-CT. Reported studies that incorporate higher energy $\mathrm{x}$ rays used non-PB-CT phase-contrast techniques. ${ }^{26,35,45,48}$ In particular, studies that utilized analyzer-based CT allow for increased sensitivity, and thus contrast resolution, at higher $\mathrm{x}$-ray energies due to extracomplicated optical elements that are not used in PB-CT. ${ }^{45,48}$

Superior contrast resolution is essential to identifying possible pathologies in the breast. The ability of PB-CT images to demonstrate a higher radiological image quality than AB-CT images is beneficial for increasing the sensitivity and specificity of breast cancer diagnosis. A strength of our study was including a range of mastectomies that contained malignant, benign, and cancerfree features, which was reflective of the diverse clinical experience.

One of the limitations of this study was that breast composition and size was not a factor when establishing the optimum energy that can produce the highest image quality. ${ }^{28} \mathrm{~A}$ denser and/or larger breast may result in a higher optimum energy, compared with breasts containing less fibroglandular tissue or being smaller, as increased penetration is required. ${ }^{48}$ There is a low

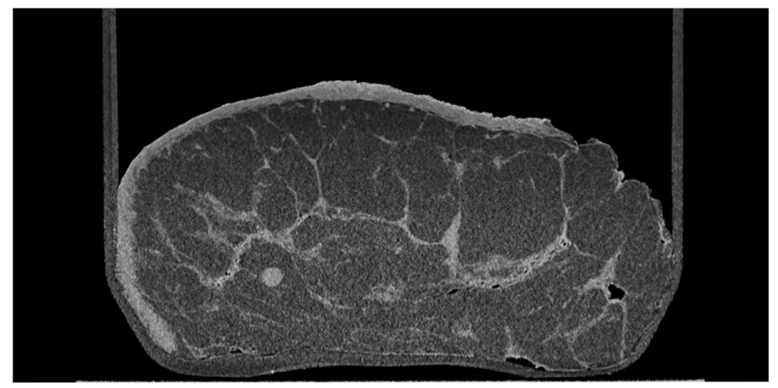

(a)

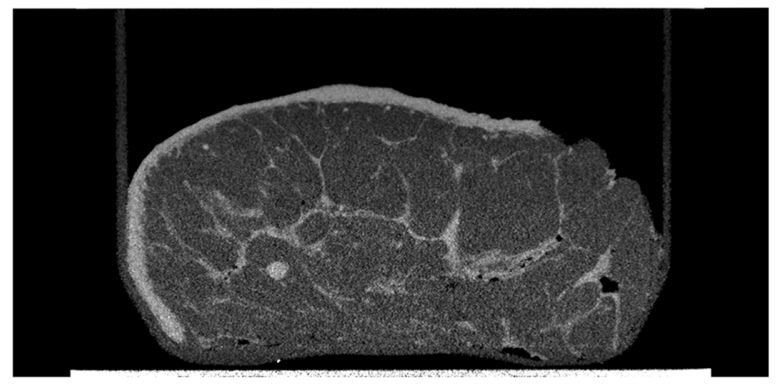

(b)

Fig. 2 Breast mastectomy with invasive lobular carcinoma and BRE grade 2 (sample ID: 323592); (a) axial PB-CT slice obtained at an x-ray energy of $32 \mathrm{keV}$ and object-to-detector distance of $6 \mathrm{~m}$; (b) axial AB-CT slice obtained at an x-ray energy of $32 \mathrm{keV}$ and nearly zero object-to-detector distance. 
specificity rate in diagnosing breast cancer in women with dense breasts [graded 3 or 4 using a Breast Imaging-Reporting and Data System (BI-RADS)] ${ }^{8,49}$ Breast density and size are factors that also differ greatly among women. ${ }^{50}$ Thus, it is important to investigate patient-specific parameters when optimizing exposure protocols in further studies to optimize PB-CT for a clinical setting.

While using seven assessors in image assessment studies is common/typical, it is difficult to generalize the results of this study to a population of assessors. In addition, as the reading order was the same for all assessors, reading-order bias could be considered another limitation in this study. To reduce this bias, the order of samples could be randomized for each observer.

Finally, $\mathrm{x}$-ray energy is only one parameter required to optimize PB-CT. Other factors including the sample-detector distance, CT reconstruction method, phase retrieval algorithm, maximum intensity projection, detector type, and pixel size need to be incorporated for effective practical implementation.

\section{Conclusions}

In this study, we evaluated the optimum x-ray energy required to achieve the PB-CT image of the highest radiological quality for mastectomy specimens. The results of this study, which utilized a Hamamatsu detector, show that an optimum range of $x$-ray energies consisting of 28, 30, 32, and $34 \mathrm{keV}$ can provide greater image quality than conventional AB-CT images. Overall, this study discovered that, under the imaging conditions used in this study, the highest radiological PB-CT image of the breast was produced by an X-ray energy of $30 \mathrm{keV}$. Future studies should consider the effect of breast density and size as well as the choice of detector and its pixel size, as these factors may change the optimum x-ray energy range. Optimizing the imaging parameters of PBCT enables the introduction of the modality into the clinical environment to improve the accuracy and efficacy of breast cancer diagnosis. The work toward clinical implementation of PB-CT for breast cancer imaging is ongoing at different synchrotron facilities. In particular, a multiinstitutional, multinational collaboration is planning the world's first patient trial of PB-CT to be conducted within the next 2 years at the IMBL of the Australian Synchrotron.

\section{Disclosures}

The authors declare no conflict of interest in this study.

\section{Acknowledgments}

The images used in this study were obtained at the Imaging and Medical beamline at the Australian Synchrotron, ANSTO. We give a special thanks to the individuals who consented to having their mastectomies used for research purposes in this study. This study was funded by the National Health and Medical Research Council, Australia (Grant No. APP1138283).

\section{References}

1. J. Ferlay et al., "Estimating the global cancer incidence and mortality in 2018: GLOBOCAN sources and methods," Int. J. Cancer 144(8), 1941-1953 (2019).

2. World Health Organisation, "Breast cancer," https://www.who.int/cancer/prevention/ diagnosis-screening/breast-cancer/en/ (accessed 3 October 2019).

3. Australian Institute of Health and Welfare, "BreastScreen Australia Monitoring Report 2020," https://www.aihw.gov.au/reports/cancer-screening/breastscreen-australia-monitoringreport-2020/contents/summary (accessed 2 May 2021).

4. S. H. Heywang-Köbrunner, A. Hacker, and S. Sedlacek, "Advantages and disadvantages of mammography screening," Breast Care 6(3), 199-207 (2011).

5. S. Ciatto et al., "Integration of 3D digital mammography with tomosynthesis for population breast-cancer screening (STORM): a prospective comparison study," Lancet Oncol. 14(7), 583-589 (2013). 
Wan et al.: Effect of $\mathrm{x}$-ray energy on the radiological image quality in propagation-based...

6. E. F. Conant et al., "Breast cancer screening using tomosynthesis in combination with digital mammography compared to digital mammography alone: a cohort study within the PROSPR consortium," Breast Cancer Res. Treat. 156(1), 109-116 (2016).

7. M. Zeeshan et al., "Diagnostic accuracy of digital mammography in the detection of breast cancer," Curēus 10(4), e2448-e2448 (2018).

8. M. T. Mandelson et al., "Breast density as a predictor of mammographic detection: comparison of interval- and screen-detected cancers," J. Natl. Cancer Inst. 92(13), 1081-1087 (2000).

9. J. Lei et al., "Diagnostic accuracy of digital breast tomosynthesis versus digital mammography for benign and malignant lesions in breasts: a meta-analysis," Eur. Radiol. 24(3), 595602 (2014).

10. X.-A. Phi et al., "Digital breast tomosynthesis for breast cancer screening and diagnosis in women with dense breasts—a systematic review and meta-analysis," BMC Cancer 18(1), 380-389 (2018).

11. M. Von Euler-Chelpin et al., "Sensitivity of screening mammography by density and texture: a cohort study from a population-based screening program in denmark," Breast Cancer Research 21(1), 111-118 (2019).

12. H. Zhao et al., "Limitations of mammography in the diagnosis of breast diseases compared with ultrasonography: a single-center retrospective analysis of 274 cases," Eur. J. Med. Res. 20(1), 49-55 (2015).

13. F. J. Gilbert, L. Tucker, and K. C. Young, "Digital breast tomosynthesis (DBT): a review of the evidence for use as a screening tool," Clin. Radiol. 71(2), 141-150 (2016).

14. J. R. Dullum, E. C. Lewis, and J. A. Mayer, "Rates and correlates of discomfort associated with mammography," Radiology 214(2), 547-552 (2000).

15. S. Suhaimi et al., "Effects of reduced compression in digital breast tomosynthesis on pain, anxiety, and image quality," Malaysian J. Med. Sci. 22(6), 40-46 (2015).

16. K. K. Lindfors et al., "Dedicated breast CT: initial clinical experience," Radiology 246(3), 725-733 (2008).

17. B. Zhao et al., "Cone beam breast $\mathrm{CT}$ with multiplanar and three dimensional visualization in differentiating breast masses compared with mammography," Eur. J. Radiol. 84(1), 48-53 (2015).

18. J. Als-Nielsen and D. McMorrow, "X-rays and their interaction with matter," in Elements of Modern X-Ray Physics, pp. 1-28, John Wiley, Chichester, West Sussex (2011).

19. J. T. Dobbins and D. J. Godfrey, "Digital x-ray tomosynthesis: current state of the art and clinical potential," Phys. Med. Biol. 48(19), R65-R106 (2003).

20. A. Momose et al., "Phase-contrast x-ray computed tomography for observing biological soft tissues," Nat. Med. 2(4), 473-475 (1996).

21. A. Momose, "Recent advances in x-ray phase imaging," Jpn. J. Appl. Phys. 44, 6355-6367 (2005).

22. R. Fitzgerald, "Phase-sensitive x-ray imaging," Phys. Today 53(7), 23-26 (2000).

23. T. Takeda et al., "Phase-contrast imaging with synchrotron $\mathrm{x}$-rays for detecting cancer lesions," Acad. Radiol. 2(9), 799-803 (1995).

24. S. Tavakoli Taba et al., "X-ray phase-contrast technology in breast imaging: principles, options, and clinical application," Am. J. Roentgenol. 211(1), 133-145 (2018).

25. S. W. Wilkins et al., "Phase-contrast imaging using polychromatic hard x-rays," Nature 384(6607), 335-338 (1996).

26. Y. Zhao et al., "High-resolution, low-dose phase contrast X-ray tomography for $3 \mathrm{~d}$ diagnosis of human breast cancers," Proc. Natl. Acad. Sci. U. S. A. 109(45), 18290-18294 (2012).

27. S. Pacilè et al., "Advantages of breast cancer visualization and characterization using synchrotron radiation phase-contrast tomography," J. Synchrotron. Radiat. 25(5), 1460-1466 (2018).

28. Y. I. Nesterets and T. E. Gureyev, "Noise propagation in X-ray phase-contrast imaging and computed tomography," J. Phys. D Appl. Phys. 47(10), 105402-105428 (2014).

29. T. E. Gureyev et al., "Investigation of the imaging quality of synchrotron-based phase-contrast mammographic tomography," J. Phys. D Appl. Phys. 47(36), 365401 (2014). 
Wan et al.: Effect of $\mathrm{x}$-ray energy on the radiological image quality in propagation-based...

30. P. Baran et al., "Optimization of propagation-based x-ray phase-contrast tomography for breast cancer imaging," Phys. Med. Biol. 62(6), 2315-2332 (2017).

31. S. Tavakoli Taba et al., "Toward improving breast cancer imaging: radiological assessment of propagation-based phase-contrast CT technology," Acad. Radiol. 26(6), e79-e89 (2019).

32. W. Huda and R. B. Abrahams, "Radiographic techniques, contrast, and noise in x-ray imaging," Am. J. Roentgenol. 204(2), W126-W131 (2015).

33. P. Oliva et al., "Experimental optimization of the energy for breast-CT with synchrotron radiation," Sci. Rep. 10(1), 17430-17442 (2020).

34. P. Delogu et al., "Optimization of the energy for breast monochromatic absorption x-ray computed tomography," Sci. Rep. 9(1), 13135-13144 (2019).

35. M. U. Ghani et al., "Low dose high energy x-ray in-line phase sensitive imaging prototype: investigation of optimal geometric conditions and design parameters," J. X-Ray Sci. Technol. 23(6), 667-682 (2015).

36. D. Paganin et al., "Simultaneous phase and amplitude extraction from a single defocused image of a homogeneous object," J. Microsc. 206(1), 33-40 (2002).

37. S. Tavakoli Taba et al., "Propagation-based phase-contrast CT of the breast demonstrates higher quality than conventional absorption-based CT even at lower radiation dose," Acad. Radiol. 28(1), e20-e26 (2021).

38. Y. I. Nesterets et al., "A feasibility study of x-ray phase-contrast mammographic tomography at the imaging and medical beamline of the australian synchrotron," J. Synchrotron. Radiat. 22(6), 1509-1523 (2015).

39. T. E. Gureyev et al., "Propagation-based x-ray phase-contrast tomography of mastectomy samples using synchrotron radiation," Med. Phys. 46(12), 5478-5487 (2019).

40. M. Båth and L. G. Månsson, "Visual grading characteristics (VGC) analysis: a non-parametric rank-invariant statistical method for image quality evaluation," Br. J. Radiol. 80(951), 169-176 (2007).

41. E. Ludewig, A. Richter, and M. Frame, "Diagnostic imaging- evaluating image quality using visual grading characteristic (VGC) Analysis," Veterinary Res. Commun. 34(5), 473-479 (2010).

42. K. McGraw and S. Wong, "Forming inferences about some intraclass correlation coefficients," Psychol. Methods 1(1), 30-46 (1996).

43. T. K. Koo and M. Y. Li, "A guideline of selecting and reporting intraclass correlation coefficients for reliability research," J Chiropr. Med. 15(2), 155-163 (2016).

44. "IBM SPSS Statistics for Windows," Version 27.0, IBM Corp., Armonk, New York.

45. P. C. Diemoz, A. Bravin, and P. Coan, "Theoretical comparison of three X-ray phase-contrast imaging techniques: propagation-based imaging, analyzer-based imaging and grating interferometry," Opt. Express 20(3), 2789-2805 (2012).

46. Hamamatsu, "X-ray source and camera selection guide," https:/www.hamamatsu.com/ resources/pdf/etd/XPRD1001E.pdf (accessed May 15, 2021).

47. A. Tomal, D. M. Cunha, and M. E. Poletti, "Optimal x-ray spectra selection in digital mammography: a semi-analytical study,” IEEE Trans. Nucl. Sci. 60(2), 728-734 (2013).

48. A. Sztrókay et al., "High-resolution breast tomography at high energy: a feasibility study of phase contrast imaging on a whole breast," Phys. Med. Biol. 57(10), 2931-2942 (2012).

49. S. Weigel et al., "Digital mammography screening: sensitivity of the programme dependent on breast density," Eur. Radiol. 27(7), 2744-2751 (2017).

50. B. L. Sprague et al., "National performance benchmarks for modern diagnostic digital mammography: update from the breast cancer surveillance consortium," Radiology 283(1), 5969 (2017).

Sarina Wan is a recent graduate of the University of Sydney's Bachelor of Applied Science (diagnostic radiography) honors class. During her final years of the course, she investigated phase-contrast imaging, a technique allowing for increased radiological quality in diagnosing breast images. She currently works in Sydney as a clinical radiographer in several modalities including mammography.

Biographies of the other authors are not available. 